\title{
Nozzle Contours for Minimum Particle-Lag Loss
}

\author{
Frank E. Marble* \\ California Institute of Technology, Pasadena, Calif.
}

\begin{abstract}
The flow of a gas-particle mixture through a rocket nozzle is analyzed under the approximation that the particle slip velocity is small compared with the average mixture velocity, using one-dimensional gasdynamics, the Stokes drag law, and corresponding approximations for the heat transfer between solid and gas phase. The variational problem defining the pressure distribution giving the minimum impulse loss due to particle lag is formulated and solved for nozzles of prescribed mass flow, length, and of given exit pressure or area. The throat section of the optimum nozzle is considerably elongated and more gradual than that of the conventional nozzle. The velocity and temperature lags were much lower (about $\frac{1}{3}$ ) in the throat region than those for the conventional nozzle. The impulse loss of the optimum nozzle was, however, reduced only about $30 \%$ below that of the conventional nozzle. It is concluded that contouring of the nozzle to improve gas-particle flow performance will result in only very modest gains. As a direct consequence, the impulse losses calculated herein for optimum nozzles can be used as a rough but convenient approximation for the impulse losses in conventional nozzles having the same area ratio or pressure ratio.
\end{abstract}

\section{Introduction}

$\mathbf{T}$ HE presence of small solid particles in the exhaust of rocket motors causes losses in specific impulse, the magnitude of which depends upon the size of the solid particles and the fraction of exhaust mass flow in solid phase. This loss arises because 1) the particle velocity lags behind the velocity of the gas, 2) heat is stored in the solid particles, and 3 ) the relative motion of gas and particles results in a dissipation that reduces the gas stagnation pressure below the chamber value. One may calculate the losses to be expected in any nozzle flow of a heterogeneous mixture to a reasonable degree of accuracy. The error is determined by the accuracy of particle drag and heat-transfer information and by some uncertainties in the transport properties of the gas. Numerical calculation of losses has been made ${ }^{1-3}$ for a variety of nozzle geometries. For small particles in nozzles that do not incorporate too abrupt gas acceleration, the problem may be simplified, assuming the lag to be a small fraction of the gas velocity. Such calculations may be carried out quite directly.

The question naturally arises concerning the shape or contour of nozzle which will minimize the particle losses. Now the particle losses are not so dominant that they dictate the nozzle shape over relatively more important considerations of heat transfer and structural weight. Rather, the optimum shape indicates the general contour to be favored when major requirements permit, and, moreover; the optimum performance provides a limit with which to compare the losses in conventional nozzles.

The general optimum problem is very difficult to handle; the linearized treatment for small lag affords a more tractable problem, and it is considered from this point of view here.

The linearized analysis of the heterogeneous flow was first published by Rannie. ${ }^{4}$ The linearization was effected assuming the differences between particle and gas velocities and temperatures to be small, taking as the unperturbed state the "equilibrium" flow where gas and particles had common velocities and temperatures. The distinguishing innovation introduced by Rannie was the utilization of gas pressure as the independent variable, determining later the axial location of any value of pressure. This transformation avoids the annoying singularity that occurs in the problem

Received June 20, 1963; revision received September 10, 1963.

* Professor of Jet Propulsion and Mechanical Engineering; also Consultant, Research Laboratories, Aeronutronic Division, Ford Motor Company, Newport Beach, Calif. Member AIAA. where the gas Mach number reaches unity. Subsequently, Marble $^{5}$ carried out a similar linearized analysis for onedimensional heterogeneous flow, employing the pressure as independent variable and assuming the axial location of each pressure value to be a prescribed function. The nozzle cross-sectional area was then calculated later as a perturbation series. Thus the analyses of Rannie and Marble constitute the linearized treatment of the "direct" and "inverse" problems, respectively, for nozzle flows containing solid particles.

In the problem of nozzle optimization, it is most convenient to start with the present author's formulation of the inverse problem. ${ }^{5}$ The problem is then posed as follows. Given the rocket chamber pressure and the nozzle exhaust pressure, what distribution of pressure along the nozzle axis will give the maximum specific impulse for a nozzle of fixed length?

\section{One-Dimensional Gasdynamics of Gas-Particle Mixture}

Denote the gas velocity parallel to the nozzle axis by $u$, the mass density by $\rho$, and designate quantities associated with particles of a single size by a subscript $p$. Equations of continuity for each phase are

$$
\begin{aligned}
\rho u A & =\dot{m} \\
\rho_{p} u_{p} A & =\varkappa \dot{m}
\end{aligned}
$$

where $\dot{m}$ is the mass flow rate of gas through a cross section $A$, and $x \dot{m}$ is the mass flow rate of solid particles. The corresponding momentum equations are

$$
\begin{gathered}
\rho u(d u / d x)+(d p / d x)=F_{p} \\
\rho_{p} u_{p}\left(d u_{p} / d x\right)=-F_{p}
\end{gathered}
$$

where $p$ is the local gas pressure, and $F_{p}$ is the force exerted upon a unit volume of gas by the particles. Assuming that the particles obey the first-order Stokes drag law, the force $F_{p}$ is

$$
F_{p}=n_{p} \cdot 6 \pi \sigma \mu\left(u_{p}-u\right)=\rho_{p} a\left[\left(u_{p}-u\right) / \lambda_{v}\right]
$$

where $n_{p}$ is the number of particles of radius $\sigma$ and mass $m$ in a unit volume, and the effective mass density $\rho_{p}$ of the solid phase is $m n_{p}$. It will be assumed that the gas viscosity $\mu \sim T^{1 / 2}$, so that the ratio $\mu / a$ is constant, where $a$ denotes the gaseous velocity of sound. The quantity $\lambda_{v}$, 
the velocity equilibration range ${ }^{5}$ of a single particle, is defined as

$$
\lambda_{v} \equiv m a / 6 \pi \sigma \mu
$$

Physically, $\lambda_{v}$ is the distance traversed by the particle in a gas moving at sonic velocity while the particle reduces its relative velocity to $e^{-1}$ of the initial value. For rocket motor atmospheres and particle radii of $1 \mu, \lambda_{v}$ is of the magnitude of a centimeter and varies as the square of particle radius.

The first law of thermodynamics for the gas is

$$
\rho u c_{p}(d T / d x)=u(d p / d x)+\left(u_{p}-u\right) F_{p}+Q_{p}
$$

where $Q_{p}$ is the heat transferred per unit volume per second from the particles to gas; the term $\left(u_{p}-u\right) F_{p}$ is the dissipative work done on the gas by particles passing through the gas. The value of $c_{p}$, specific heat of the gas at constant pressure, is assumed constant. To the same approximation of Stokes law, the heat transfer rate $Q_{p}$ from particles to gas may be written as

$$
Q_{p}=n_{p}(k / \sigma) 4 \pi \sigma^{2}\left(T_{p}-T\right) \equiv \rho_{p} c_{p} a\left[\left(T_{p}-T\right) / \lambda_{T}\right]
$$

where $k$ is the thermal conductivity of the gas, and $T_{p}$ is the local temperature of particles. The temperature equilibration range $\lambda_{T}$ has a physical significance similar to its counterpart $\lambda_{v}$ and is defined as

$$
\lambda_{T} \equiv c_{p} m a / 4 \pi \sigma k=\frac{3}{2} \operatorname{Pr} \lambda_{v}
$$

Usually, the value of the Prandtl number, $\operatorname{Pr} \equiv c_{p} \mu / k$, is assumed to be a constant and is near enough to $\frac{2}{3}$ that $\lambda_{v}$ and $\lambda_{T}$ may be considered equal.

The first law of thermodynamics for the solid phase is

$$
\rho_{p} u_{p} c\left(d T_{p} / d x\right)=-Q_{p}
$$

where $c$ is the constant specific heat of the solid material, and the individual solid particles are assumed undeformed by stresses imposed upon them by the gas.

Together with the gaseous equation of state, the preceding relations give a complete analytical description of the onedimensional heterogeneous flow. Because the differences $u-u_{p}$ and $T-T_{p}$ will be considered small in comparison with $u$ and $T$, respectively, the following variables will prove convenient:

$$
\begin{gathered}
u-u_{p} \equiv u_{s} \\
T-T_{p} \equiv T, \\
1-\left(\rho_{p} / \varkappa \rho\right) \equiv \rho_{s}
\end{gathered}
$$

A transformation in the equations of continuity, momentum, and energy may be effected to emphasize the fact that $u_{s}, T_{s}$, and $\rho_{s}$ are essentially of much smaller numerical magnitude than $u, T$, and unity, respectively. The force $F_{p}$ acting between the two phases may be eliminated from Eqs. (3) and (4) to give

$$
(1+\varkappa) \rho u \frac{d u}{d x}+\frac{d p}{d x}=\varkappa \rho u \frac{d u}{d x}
$$

In much the same manner, the heat exchange between the two phases may be eliminated from Eqs. (7) and (9) to yield a relation that may be integrated from the rocket chamber to an arbitrary position of the nozzle:

$$
\left(\frac{c_{p}+\varkappa c}{1+\varkappa}\right)\left(T-T_{c}\right)+\frac{1}{2} u^{2}=\frac{\varkappa}{1+\varkappa}\left[c T_{s}+u u_{s}-\frac{1}{2} u_{*}^{2}\right]
$$

It has been assumed that $u, u_{s}$, and $T s$ vanish in the rocket chamber and that $T_{c}$ is the common temperature of the gas and solid phases in the chamber.

Designate by "equilibrium flow" the limiting circumstance where the velocities and temperatures of the two phases remain exactly equal throughout the nozzle, that is, where $\rho_{0}=u_{*}=T_{*}=0$. The flow is then described by

$$
\begin{gathered}
(1+\varkappa) \rho u \frac{d u}{d x}+\frac{d p}{d x}=0 \\
\left(\frac{c_{p}+\varkappa c}{1+\varkappa}\right)\left(T-T_{c}\right)+\frac{1}{2} u^{2}=0 \\
(1+\varkappa) \rho u A=(1+\varkappa) \dot{m} \\
p=(1+\varkappa) \rho\left(\frac{R}{1+\varkappa}\right) T
\end{gathered}
$$

Equations (14-17) describe the isentropic flow of a gas through a nozzle of mass flow $(1+x) \dot{m}$ and cross-sectional area $A$, where the gas has an effective density

$$
\bar{\rho}=(1+x) \rho
$$

and effective gas properties

$$
\bar{c}_{p} \equiv\left(c_{p}+\varkappa c\right) /(1+\varkappa) \quad \vec{R} \equiv\left(c_{p}-c_{v}\right) /(1+\varkappa)
$$

The expansion process takes place according to the law

$$
\frac{T}{T_{c}}=\left(\frac{p}{p_{c}}\right)^{(\bar{\gamma}-1) / \bar{\gamma}}=\left(\frac{\bar{\rho}}{\bar{\rho}_{c}}\right)^{\bar{\gamma}-1}
$$

where $p_{c}$ and $\bar{\rho}_{c}$ are the pressure and effective density in the rocket chamber, and $\bar{\gamma}=\bar{c}_{p} / \bar{c}_{\boldsymbol{v}}$. All familiar relationships for isentropic nozzle flow hold in terms of these effective quantities.

Returning now to the problem of heterogeneous nozzle flow under nonequilibrium conditions, a relation resembling the isentropic integral, Eq. (20), may be obtained from Eqs. (12) and (13), together with appropriate equations of state and continuity:

$$
\begin{array}{r}
\left(\frac{T}{T_{c}}\right)\left(\frac{p_{c}}{p}\right)^{(\bar{\gamma}-1) / \bar{\gamma}}=\exp \left\{\frac { \varkappa } { 1 + \varkappa } \int _ { 0 } ^ { \times } \frac { 1 } { \overline { c } _ { p } T } \left[c \frac{d T_{\bullet}}{d x}+\right.\right. \\
\left.\left.u_{s} \frac{d}{d x}\left(u-u_{\bullet}\right)\right] d x\right\}
\end{array}
$$

In the analysis of nonequilibrium heterogeneous flow, it will be convenient to work with Eqs. (13) and (21), but they do not yet suffice to complete the problem. From Eqs. (1) and (2), it follows that

$$
\rho_{s} u+u_{s}=\rho_{s} u \text {. }
$$

whereas from Eqs. (4) and (1) it is not difficult to show

$$
u \frac{d u}{d p}-\frac{a u_{*}}{\bar{\lambda}_{v}} \frac{1}{d p / d x}=-\rho_{s} \frac{a u_{*}}{\bar{\lambda}_{v}} \frac{1}{d p / d x}+u \frac{d u_{s}}{d p}
$$

where $a$ is the equilibrium speed of sound, $a^{2}=\bar{\gamma} \bar{R} T$, and $\bar{\lambda}_{v}$ is the velocity equilibration length [Eq. (6)] based upon the equilibrium speed of sound. Similarly, from Eqs. (10) and (11),

$$
u \frac{d T}{d p}-\frac{\bar{c}_{p}}{c} \frac{a T}{\bar{\lambda}_{T}} \frac{1}{d p / d x}=-\rho_{s} \frac{\bar{c}_{p}}{c} \frac{a T \cdot}{\bar{\lambda}_{T}} \frac{1}{d p / d x}+u \frac{d T}{d p}
$$

where $\bar{\lambda}_{T}$ is based upon the equilibrium speed of sound and specific heat $\bar{c}_{p}$. The set of equations $(13,16,17$, and $21-$ 24 ) is completely equivalent to the original relations, and these equations are written explicitly in terms of the quantities $u_{s}, T_{s}$, and $\rho_{s}$. Moreover, the independent variable has been changed to the gas pressure $p$, and the distribution of pressure along the nozzle axis, $p(x)$ or its inverse, is assumed to be prescribed.

\section{Solution for Flow with Small Slip}

When the three dependent variables $\rho_{s}, u_{s}$, and $T$, are small, a perturbation solution naturally suggests itself, and the 
appropriate small quantity in terms of which the solutions should be expanded is $\bar{\lambda}_{v} / L$, where $L$ is the fixed nozzle length. The state of the gas may then be written as

$$
\begin{aligned}
& \rho=\rho^{(0)}+\left(\bar{\lambda}_{v} / L\right) \rho^{(1)}+\left(\bar{\lambda}_{v} / L\right)^{2} \rho^{(2)}+\ldots \\
& u=u^{(0)}+\left(\bar{\lambda}_{v} / L\right) u^{(1)}+\left(\bar{\lambda}_{v} / L\right)^{2} u^{(2)}+\ldots \\
& T=T^{(0)}+\left(\bar{\lambda}_{v} / L\right) T^{(1)}+\left(\bar{\lambda}_{v} / L\right)^{2} T^{(2)}+\ldots
\end{aligned}
$$

where each coefficient is a function of the local pressure $p$. Each of these variables has a nonvanishing zeroth degree part, and all coefficients in the expansions are of order unity. The variations of the particle state from that of the gas, $\rho_{s}, u_{s}$, and $T_{s}$, have leading terms of the first degree:

$$
\begin{aligned}
& \rho_{s}=\left(\bar{\lambda}_{v} / L\right) \rho_{s}{ }^{(1)}+\left(\bar{\lambda}_{v} / L\right)^{2} \rho_{s}{ }^{(2)}+\ldots \\
& u_{s}=\left(\bar{\lambda}_{v} / L\right) u_{s}{ }^{(1)}+\left(\bar{\lambda}_{v} / L\right)^{2} u_{s}{ }^{(2)}+\ldots \\
& T_{s}=\left(\bar{\lambda}_{v} / L\right) T_{s}{ }^{(1)}+\left(\bar{\lambda}_{v} / L\right)^{2} T_{s}{ }^{(2)}+\ldots
\end{aligned}
$$

The functions giving terms of various order in each variable may be determined by substituting expressions (25) and (26) into the Eqs. (13, 16, 17, and 21-24) and separating each equation according to powers of the small parameter $\bar{\lambda}_{v} / L$.

The functions $\rho^{(0)}, u^{(0)}$, and $T^{(0)}$ are described by the zeroth order parts of equations $(13,17$, and 21$)$, and they correspond exactly to the equilibrium solution described by equations $(15,17$, and 20). To the zeroth order of approximation, the gas and particles have the same velocity and temperature, and the solution being employed consists in a perturbation expansion about the "equilibrium" flow. Physically, this implies that, as $\bar{\lambda}_{v} / L$ becomes very small, the actual flow approaches more and more closely to the equilibrium flow. That is equivalent to saying that, when the equilibration range $\bar{\lambda}_{v}$ is negligible compared with the nozzle length, the gas and particles achieve a local equilibrium state before a significant fraction of the nozzle length has been traversed. The equilibrium solution is readily written down in terms of the prescribed pressure distribution along the nozzle:

$$
\begin{gathered}
\frac{\rho^{(0)}}{\rho_{c}}=\left(\frac{p}{p_{c}}\right)^{1 / \bar{\gamma}} \\
\frac{T^{(0)}}{T_{c}}=\left(\frac{p}{p_{c}}\right)^{(\bar{\gamma}-1) / \bar{\gamma}} \\
u^{(0)}=\left\{2 \bar{c}_{p} T_{c}\left[1-\left(\frac{p}{p_{c}}\right)^{(\bar{\gamma}-1) / \bar{\gamma}}\right]\right\}^{1 / 2}
\end{gathered}
$$

Calculation of the first-order terms $\rho_{s}^{(1)}, u_{s}^{(1)}$, and $T_{s}^{(1)}$ follows quite directly from Eqs. (22-24). From the zerothorder part of Eq. (23), it follows that

$$
u_{s}^{(1)}=-\frac{a^{(0)}}{\bar{\gamma}}\left(\frac{1}{p} \frac{d p}{d \xi}\right)
$$

where the new length variable $\xi=x / L$ has been introduced. Similarly, from the form of the energy relation given by Eq. (24), it follows, after some reduction, that

$$
T_{s}^{(1)}=\frac{1}{\bar{c}_{p}} \frac{c}{\bar{c}_{p}}\left(\frac{\lambda_{T}}{\bar{\lambda}_{v}}\right) \frac{a^{(0)}}{\bar{\gamma}} u^{(0)}\left(\frac{1}{p} \frac{d p}{d \xi}\right)
$$

and, from Eq. (22),

$$
\rho_{s}^{(1)}=\frac{1}{\bar{\gamma}} \frac{1}{M^{(0)}}\left(\frac{1}{p} \frac{d p}{d \xi}\right)
$$

From Eqs. (30) and (31), in which $u_{a}^{(1)}$ and $T_{c}^{(1)}$ are determined algebraically, it is apparent that the order of differential equations has been reduced by one in each case; that is, the perturbation employed is a singular one.

Evaluation of the remaining first-order terms may be carried out from Eqs. (13, 17, and 21), noting particularly the fact that the right-hand sides of both Eqs. (13) and (21) are of the first order and are known to that order. These two perturbation relations may be written explicitly as

$$
\frac{T^{(1)}}{T^{(0)}}+(\bar{\lambda}-1) M^{(0) 2}\left(\frac{u^{(1)}}{u^{(0)}}\right)=\frac{\varkappa}{1+\varkappa} F\left(\frac{p}{p_{c}}\right)
$$

and

$$
\frac{T^{(1)}}{T^{(0)}}=\frac{\varkappa}{1+\varkappa} G\left(\frac{p}{p_{c}}\right)
$$

where the functions on the right-hand sides of Eqs. (33) and (34) are the known functions of the prescribed pressure ratio

$$
F\left(\frac{p}{p_{c}}\right)=(\eta-1)\left(\frac{\bar{\gamma}-1}{\bar{\gamma}}\right) M^{(0)} \frac{1}{p} \frac{d p}{d \xi}
$$

and

$$
G\left(\frac{p}{p_{c}}\right)=\int_{p_{0}}^{p} \frac{1}{\bar{c}_{p} T^{(0)}}\left[(1-\eta) u_{s}^{(1)} \frac{d u^{(0)}}{d p}-\eta u^{(0)} \frac{d u_{s}^{(1)}}{d p}\right] d p
$$

Here, the notation $\left(\bar{\lambda}_{T} / \bar{\lambda}_{v}\right)\left(c / \bar{c}_{p}\right)^{2} \equiv \eta$ has been adopted. The first-order perturbation to the equation of state, Eq. (17), gives

$$
\rho^{(1)} / \rho^{(0)}=-T^{(1)} / T^{(0)}
$$

Now Eq. (34) gives directly the first-order gas temperature perturbation, and Eqs. (33) and (37) may be solved for velocity and density perturbations directly as

$$
\frac{u^{(1)}}{u^{(0)}}=\frac{\varkappa}{1+\varkappa} \frac{1}{(\bar{\gamma}-1) M^{(0) 2}} F\left(\frac{p}{p_{c}}\right)-G\left(\frac{p}{p_{c}}\right)
$$

and

$$
\frac{\rho^{(1)}}{\rho^{(0)}}=-\frac{\varkappa}{1+\varkappa} G\left(\frac{p}{p_{c}}\right)
$$

The first-order modifications to equilibrium nozzle flow, resulting from the effects of solid particles transported by the gas, are then given by Eqs. (34, 38, and 39) for the gas flow and Eqs. $(30-32)$ for the particle flow where the gas pressure is a prescribed function of $x$ or of $\xi$.

The required cross-sectional area of a nozzle carrying mass flow $\dot{m}$ and providing the required pressure distribution may be expressed as

$$
A\left(\frac{p}{p_{c}}\right)=A^{(0)}\left(\frac{p_{c}}{p}\right)+\frac{\lambda_{v}}{L} A^{(1)}\left(\frac{p}{p_{c}}\right)+\ldots
$$

where the coefficients $A^{(0)}, A^{(1)}$, etc., are readily determined from the equation of continuity, Eq. (1). The area $A^{(0)}$ for equilibrium flow may be expressed by the parameter $\rho_{c} a_{c} A^{(0)} / \dot{m}$ :

$\frac{\rho_{c} a_{c} A^{(0)}}{\dot{m}}\left(\frac{p}{p_{c}}\right)=\left(\frac{p}{p_{c}}\right)^{1 / \bar{\gamma}}\left\{\frac{2}{\bar{\gamma}-1}\left[1-\left(\frac{p}{p_{c}}\right)^{(\bar{\gamma}-1) / \bar{\gamma}}\right]\right\}^{-1 / 2}$

since the chamber conditions are assumed constant and the area may be scaled according to mass flow. The ratio of the first area perturbation to the zeroth-order area is found from the continuity equation and expressed in terms of the $F$ and $G$ functions. Through partial integration of (36) and ensuing simplification, the expression for $A^{(1)} / A^{(0)}$ may be written as

$$
\begin{aligned}
& \frac{A^{(1)}}{A^{(0)}}=\frac{\varkappa}{1+\kappa} \frac{1}{\bar{\gamma} M^{(0)}}\left\{\left[1+\eta(\bar{\gamma}-1) M^{(0) 2}\right] \frac{1}{p} \frac{d p}{d \xi}+\right. \\
& \left.\quad \frac{1+(\bar{\gamma}-1) M^{(0) 2}}{\bar{\gamma}^{2} \bar{M}^{(0) 2}} \int_{1}^{\mathrm{p} / \mathrm{p}_{\mathrm{o}}} \frac{1+\eta(\bar{\gamma}-1) M^{(0) 2}}{M^{(0)}} \frac{1}{\alpha^{2}} \frac{d \alpha}{d \xi} d \alpha\right\}
\end{aligned}
$$

None of these expressions encounter difficulties in the neighborhood of the nozzle throat. 


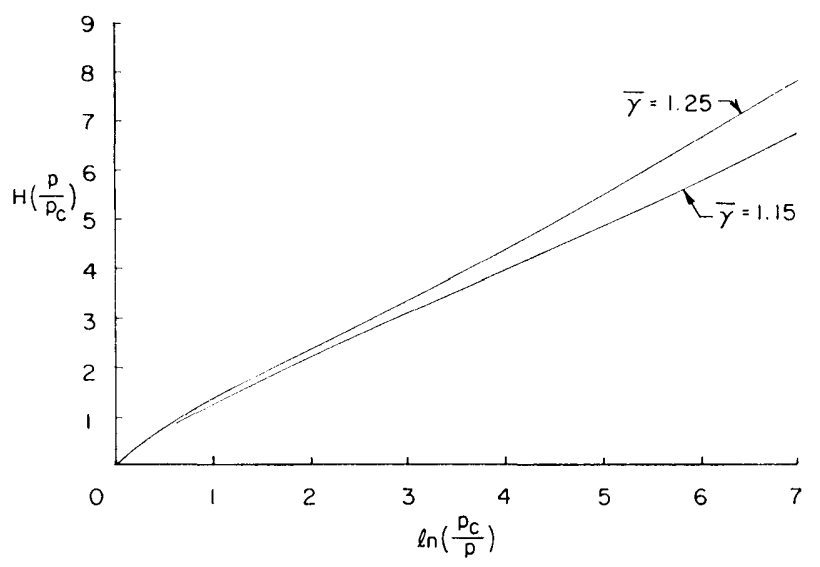

Fig. 1 Values of the function $H\left(p / p_{c}\right)$ as a function of pressure ratio $(\bar{\gamma}=1.25,1.15 ; \eta=1)$.

\section{Nozzle for Minimum Loss in Specific Impulse}

To calculate the specific impulse loss to the first order in $\lambda_{v} / L$, consider a nozzle of fixed length $L$ expanding the gas from a given chamber state $p_{c}, T_{c}$ to a prescribed exhaust pressure $p_{e}$. If the specific impulse

$$
I^{(0)} \equiv u_{e}^{(0)} / g
$$

occurring under conditions of equilibrium flow is taken as the reference value, then the fractional loss of impulse caused by the presence of the particles is

$$
\frac{I^{(0)}-I}{I^{(0)}}=-\frac{\lambda_{v}}{L}\left[\frac{u^{(1)}}{u^{(0)}}-\frac{\varkappa}{1+\varkappa} \frac{u_{s}^{(1)}}{u^{(0)}}\right]_{p_{e}}
$$

where, since the pressure distribution is prescribed, nozzles carrying both equilibrium and nonequilibrium flow are expanded to $p_{e}$, the local atmospheric pressure. This loss may be written down explicitly using the values for $u^{(1)} / u^{(0)}$ and $u_{s}{ }^{(1)} / u^{(0)}$ from Eqs. (38) and (30), respectively. With this substitution,

$$
\begin{aligned}
\frac{I^{(0)}-I}{I^{(0)}}= & \frac{\lambda_{v}}{L} \frac{\varkappa}{1+\varkappa}\left[-\frac{\eta}{\bar{\gamma} M^{(0)}} \frac{1}{p_{e}} \frac{d p}{d \xi}\left(p_{e}\right)+\right. \\
& \frac{1-\eta}{\bar{\gamma}^{2} M_{e}^{(0) 2}} \int_{\mathrm{p}_{\mathrm{e}}}^{\mathrm{p}_{\mathrm{e}}} \frac{1}{M^{(0)}} \frac{1}{p^{2}} \frac{d p}{d \xi} d \xi+ \\
& \left.\frac{\eta}{(\bar{\gamma}-1) M_{e}^{(0) 2}} \int_{\mathrm{p}_{\mathrm{c}}}^{\mathrm{p}_{\mathrm{e}}} \frac{u^{(0)}}{\bar{c}_{p} T^{(0)}} \frac{d}{d p}\left(\frac{a^{(0)}}{\bar{\gamma} p} \frac{d p}{d \xi}\right) d p\right]
\end{aligned}
$$

where the subscript $e$ has been used to denote the value of the

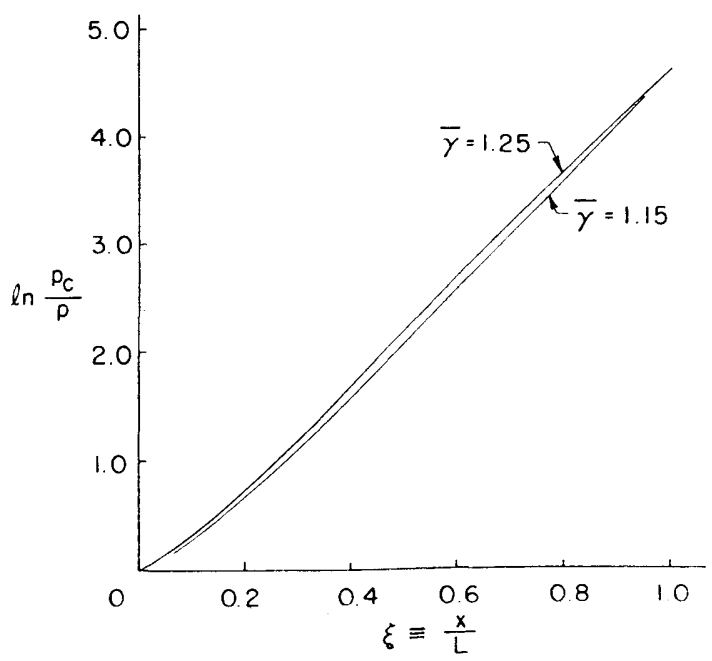

Fig. 2 Pressure distribution along nozzle of optimum contour $(\bar{\gamma}=1.25,1.15 ; \eta=1)$. variables at the nozzle exit, that is, when $p=p_{\ell}$. Partial integration of the second integral in Eq. (45) and some subsequent simplification yield a final convenient form for the fractional impulse loss:

$$
\begin{aligned}
\frac{I^{(0)}-I}{I^{(0)}}=-\frac{\bar{\lambda}_{v}}{L}\left(\frac{\varkappa}{1+\varkappa}\right) \frac{1}{\bar{\gamma}^{2} M_{e}{ }^{(0) 2}} \times \\
\quad \int_{\mathrm{p}_{0}}^{\mathrm{p}_{\mathrm{e}}} \frac{1+\eta(\bar{\gamma}-1) M^{(0) 2}}{M^{(0)}} \frac{1}{p^{2}} \frac{d p}{d \xi} d p
\end{aligned}
$$

For most cases of practical interest, $\eta \approx 1$, and the error introduced into Eq. (46) by setting $\eta=1$ is correspondingly small. Writing the Mach number in terms of the pressure,

$$
M^{(0) 2}=\frac{2}{\bar{\gamma}-1}\left[\left(\frac{p_{c}}{p}\right)^{(\bar{\gamma}-1) / \hat{\gamma}}-1\right]
$$

evaluation of Eq. (46) is, at worst, an elementary numerical integration so long as the pressure distribution along the axis $p(\xi)$, or its inverse $\xi(p)$, is prescribed.

Now Eq. (46) may be written as

$$
\frac{I^{(0)}-I}{I^{(0)}}=-C \int_{p e}^{p c} \phi\left[p, \xi(p), \frac{d \xi}{d p}\right] d p
$$

and is of the general form appropriate for treatment by the techniques of elementary variational methods. Consider the

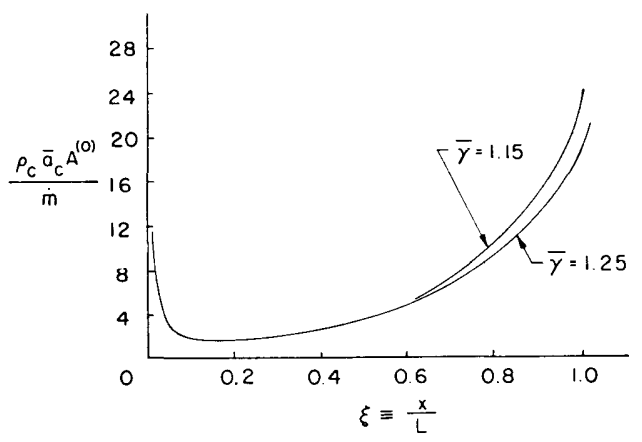

Fig. 3 Area distribution along nozzle of optimum contour $(\bar{\gamma}=1.25,1.15 ; \eta=1)$.

pressure distribution $\xi(p)$ as unknown, and ask for the distribution function $\xi(p)$ that leads to the minimum impulse loss for the expansion ratio $p_{c} / p_{e}$ subject to the condition that the nozzle be of length $L$ or, what is the same, $\xi=1$. Therefore, the distribution functions are subject to the fixed end conditions $\xi\left(p_{c}\right)=0$ and $\xi\left(p_{e}\right)=1$. Since the functional $\phi[p, \xi(p), d \xi / d p]$ is in fact

$$
\frac{1+\eta \cdot(\bar{\gamma}-1) M_{0}^{2}}{M^{(0)} p^{2}}\left(\frac{1}{d \xi / d p}\right)
$$

and consequently does not involve $\xi(p)$ but only $d \xi / d p$, the Euler equation is just

$-\frac{d}{d p} \frac{\partial \phi}{\partial(d \xi / d p)}=\frac{d}{d p}\left[\frac{1+\eta(\bar{\gamma}-1) \bar{M}_{0}^{2}}{M^{(0)} p^{2}} \frac{1}{(d \xi / d p)^{2}}\right]=0$

The solution for $\xi(p)$ may then be given in the form

$$
\xi\left(\frac{p}{p_{c}}\right)=\frac{H\left(p / p_{c}\right)}{H\left(p_{e} / p_{c}\right)}
$$

where the function $H\left(p / p_{c}\right)$ is defined by the integral

$$
H\left(\frac{p}{p_{c}}\right)=\int_{p / p c}^{1}\left[\frac{1+\eta(\bar{\gamma}-1) M^{(0) 2}}{M^{(0)}}\right]^{1 / 2} \frac{d p}{p}
$$

and the relation between $M^{(0)}$ and $p / p_{c}$ is given by Eq. (47). The integral, which plays a central role in the following optimum nozzle theory, may be written in terms of either $M^{(0)}$ 
alone or $p / p_{c}$ alone. The former choice leads to

$$
H\left(M^{(0)}\right)=\bar{\gamma} \int_{0}^{M(0)} \frac{\left\{M\left[1+\eta(\bar{\gamma}-1) M^{2}\right]\right\}^{1 / 2}}{1+[(\bar{\gamma}-1) / 2] M^{2}} d M
$$

and may be evaluated in terms of incomplete elliptic integrals of the second and third kinds. This function is shown in Fig. 1 for $\bar{\gamma}=1.25$ and $\bar{\gamma}=1.15$, where $\eta=1$. The independent variable $\ln \left(p_{c} / p\right)$ is a convenient one and will be used in succeeding representations. With $H\left(p / p_{c}\right)$ known, determination of $\xi\left(p / p_{c}\right)$, the fractional nozzle length at which any value $p / p_{c}$ of the pressure ratio occurs, is a simple matter and is shown in Fig. 2. The statement of the problem fixes the two end points of these curves, and consequently variations in $\xi\left(p / p_{c}\right)$ due to changes in $\bar{\gamma}$ are quite small. This particular nozzle shape is calculated for a pressure ratio $p_{c} / p_{c}=100$.

To the zeroth order in $\lambda_{v} / L$, the nozzle cross-sectional area parameter $\left(\rho_{c} \bar{a}_{c} A^{(0)}\right) / \dot{m}$ is given by Eq. (41) in terms of the local pressure $p / p_{c}$. Using the result of Fig. 2, one may obtain from Eq. (41) the distribution of nozzle area with length; this is shown in Fig. 3, again for a pressure ratio $p_{c} / p_{c}=$ 100 and the two values of $\bar{\gamma}$ chosen previously. The characteristics of the optimum shape then become quite clear. The contraction from the chamber (infinite area) is initially quite rapid; the throat region is greatly prolonged to reduce the usually high accelerations, and a reasonable degree of acceleration persists to the end of the nozzle. The fact that the optimum nozzle does not show small accelerations (and hence small particle slip) at the nozzle exit shows that losses due to high slip within the nozzle are as important as actual particle slip. In other words, the acceleration is distributed so that both internal losses and particle slip losses at the exit are moderately small rather than having either one particularly small and the other correspondingly large. It is also clear that the optimum nozzle contour arrived at is not an especially appealing one from the standpoints of heat transfer at the throat or divergence losses at the nozzle exit. This observation merely serves to emphasize the statement made earlier that the optimum nozzle provides a standard of comparison for particle losses in other nozzles and indicates the general features that would be incorporated when other design requirements permit.

Referring back to Eqs. (30) and (31), it is a relatively simple matter to calculate the differences in velocity and temperature between particles and gas for the optimum nozzle. For the value of $(1 / p)(d p / d \xi)$ follows directly from Eq. (51) as

$$
\frac{1}{p} \frac{d p}{d \xi}=-H\left(\frac{p_{e}}{p_{c}}\right)\left[\frac{M^{(0)}}{1+\eta(\gamma-1) M^{(0)}}\right]^{1 / 2}
$$

and a little calculation gives the slip velocity

$$
\begin{aligned}
\frac{u_{s}^{(1)}}{\bar{a}_{c}} & =\frac{1}{\bar{\gamma}} H\left(\frac{p_{e}}{p_{c}}\right) \times \\
& {\left[\frac{M^{(0)}}{\left[1+\eta(\bar{\gamma}-1) M^{(0) 2}\right]\left\{1+[(\bar{\gamma}-1) / 2] M^{(0) 2}\right\}}\right]^{1 / 2} }
\end{aligned}
$$

and the temperature difference

$$
\begin{aligned}
\frac{T_{s}^{(1)}}{T_{c}}=\eta \frac{\bar{c}_{p}}{c}\left(\frac{\bar{\gamma}-1}{\bar{\gamma}}\right) H\left(\frac{p_{e}}{p_{c}}\right) \frac{M^{(0)}}{1+[(\bar{\gamma}-1) / 2] M^{(0) 2}} \times \\
\\
{\left[\frac{M^{(0)}}{1+\eta(\bar{\gamma}-1) M^{(0) 2}}\right]^{1 / 2} }
\end{aligned}
$$

These velocity and temperature differences are shown in Figs. 4 and 5 , respectively, for the two values of $\bar{\gamma}$ used previously. Again the calculations were made for a pressure ratio $p_{c} / p_{e}=100$. The slip velocity and, to a considerable extent, the temperature difference tend to rise quickly during the early stages of expansion and to maintain this value relatively consistently throughout the nozzle. In particular,

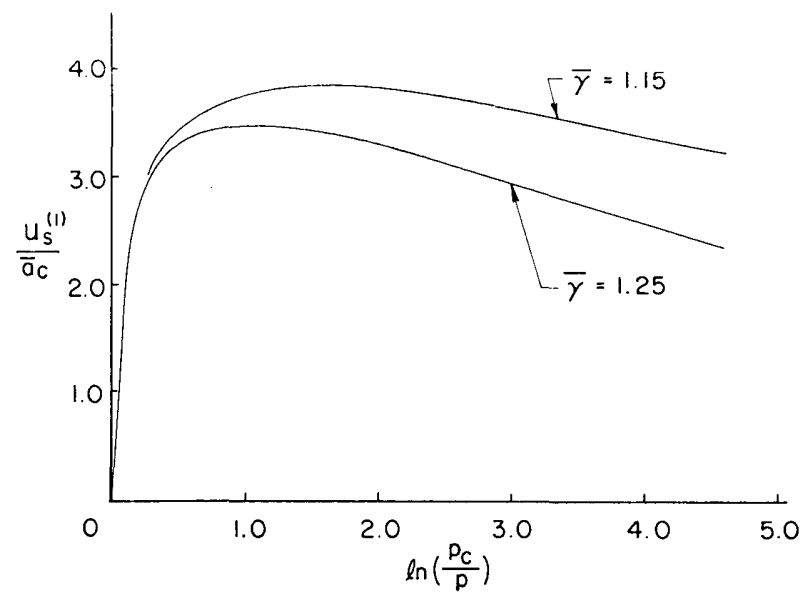

Fig. 4 Particle slip velocity given as a function of pressure ratio from chamber to a particular location along nozzle of optimum contour $(\bar{\gamma}=1.25 ; 1.15 ; \eta=1)$.

there is no strong tendency for the slip velocity or the temperature difference to approach small values at the nozzle outlet. The actual values of slip velocity and temperature difference ratios are obtained, as follows from Eq. (25), from the values appearing in Figs. 4 and 5 through multiplication by the appropriate value of $\lambda / L$, a factor usually of the order of 0.1 or less. Numerical values of the slip velocity and temperature difference are therefore at most a few percent of the $\bar{a}_{c}$ and the chamber temperature, respectively.

The first-order perturbation to the optimum nozzle shape, to correct it for the effects of particle and temperature lag, is given through Eq. (42). It will be noted in particular that the integral involved in that equation is exactly the integral that has been minimized in connection with the specific impulse calculation. Therefore, for the optimum nozzle, the area perturbation is given explicitly as

$$
\begin{gathered}
\frac{A^{(1)}}{A^{(0)}}=-\frac{\varkappa}{1+x} \frac{1}{\bar{\gamma}} H\left(\frac{p_{e}}{p_{c}}\right)\left\{\left[\frac{1+\eta(\bar{\gamma}-1) M^{(0) 2}}{M^{(0)}}\right]^{1 / 2}+\right. \\
\left.\frac{1}{\bar{\gamma} M^{(0)}}\left[\frac{1+\eta(\bar{\gamma}-1) M^{(0) 2}}{M^{(0)}}\right] H\left(\frac{p}{p_{c}}\right)\right\}
\end{gathered}
$$

Since the terms in Eq. (57) are all positive, the first-order correction tends to decrease the nozzle cross-sectional area slightly over that which would be required if the gas and particles were completely in equilibrium. This correction, shown in Fig. 6 for two values of the specific heat ratio, is nearly constant and very small over the major portion of the nozzle length. The perturbation is infinite at the chamber end of the nozzle where the zeroth-order nozzle area itself

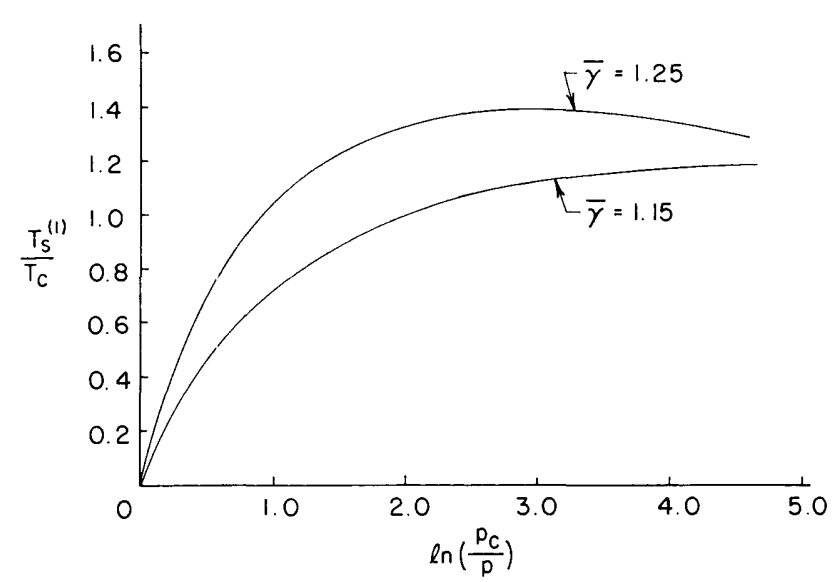

Fig. 5 Particle temperature lag as a function of pressure ratio from chamber to a particular location along nozzle of optimum contour $(\bar{\gamma}=1.25,1.15 ; \eta=1)$. 


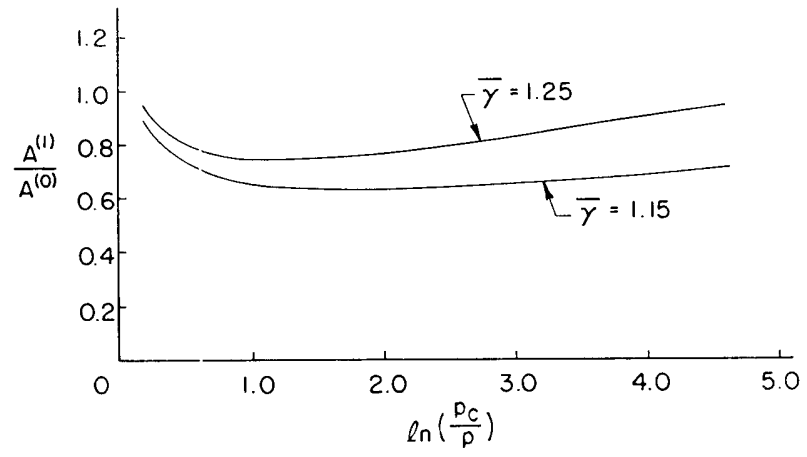

Fig. 6 First-order perturbation to cross-sectional area distribution of optimum contour nozzle $(\bar{\gamma}=1.25,1.15$; $\eta=1)$.

is infinite, because of the choice to make true stagnation conditions in the chamber. There is nothing physically unacceptable about this behavior of the area perturbation. The significant result is that the nozzle shape is maintained, but the cross-sectional area is slightly decreased roughly in proportion to the area for equilibrium flow.

The fractional loss in specific impulse due to the particle effects, given by Eq. (46), may be written also for the optimum nozzle by inserting the appropriate value of $d p / d \xi$ from Eq. (54). Then the impulse loss is

$$
\begin{aligned}
& \frac{I^{(0)}-I}{I^{(0)}}=\frac{\bar{\lambda}_{v}}{L}\left(\frac{\varkappa}{1+\varkappa}\right) \frac{1}{\bar{\gamma}^{2} M_{e}^{(0) 2}} H\left(\frac{p_{e}}{p_{c}}\right) \times \\
& \int_{p e}^{p c} \times\left[\frac{1+\eta(\bar{\gamma}-1) M^{(0) 2}}{M^{(0)}}\right]^{1 / 2} \frac{d p}{p} \\
&=\frac{\bar{\lambda}_{v}}{L}\left(\frac{\varkappa}{1+\varkappa}\right)\left[\frac{H\left(p_{e} / p_{c}\right)}{\bar{\gamma} M_{e}{ }^{(0)}}\right]^{2}
\end{aligned}
$$

where the remaining integral is recognized as that given by Eq. (52). Here $M_{e}{ }^{(0)}$ is understood to be the Mach number, based upon equilibrium flow, obtained by expanding from the chamber pressure $p_{c}$ to the prescribed exit pressure $p_{e}$. This fractional impulse loss is shown in Fig. 7 for two values of the specific heat ratio. It should be noted that the ordinate in this figure is $\ln \left(p_{c} / p_{e}\right)$, so that any ordinate refers to a specific nozzle having that expansion ratio, and the corresponding abscissa is the fractional impulse loss for that nozzle. It is convenient to note that for usual nozzles the abscissa is of the order of unity, and therefore the impulse loss $\left(I^{(0)}-I\right) / I^{(0)} \sim[\varkappa /(1+\varkappa)]\left(\lambda_{v} / L\right)$. The effect of specific heat ratio is not large, even at expansion ratios of 100 .

\section{Comparison between Optimum and Conventional Nozzles}

One of the principal interests in the optimum nozzle is the determination of just how much of the particle lag loss in a conventional nozzle can be regained by proper contouring of the nozzle. This question really resolves to deciding whether the extremal that has been found is a "sharp" one, that is, how severe a performance penalty is to be paid for departing from the optimum shape. The most straightforward and illuminating manner of investigating this sensitivity is to compare the specific impulse losses in conventional and optimum nozzles having the same length and the same prescribed pressure ratios.

The conventional shape chosen for the comparison was that used by Rannie ${ }^{4}$ in his illustrative example of particle lag calculations. This contour is constructed from three circular arcs, and the details are given in his paper. The two contours (based upon equilibrium flow) are compared in Fig. 8 , where they are scaled to the same pressure ratio as that used by Rannie; since this contour is based upon equilibrium flow, the outlet areas coincide when the throat

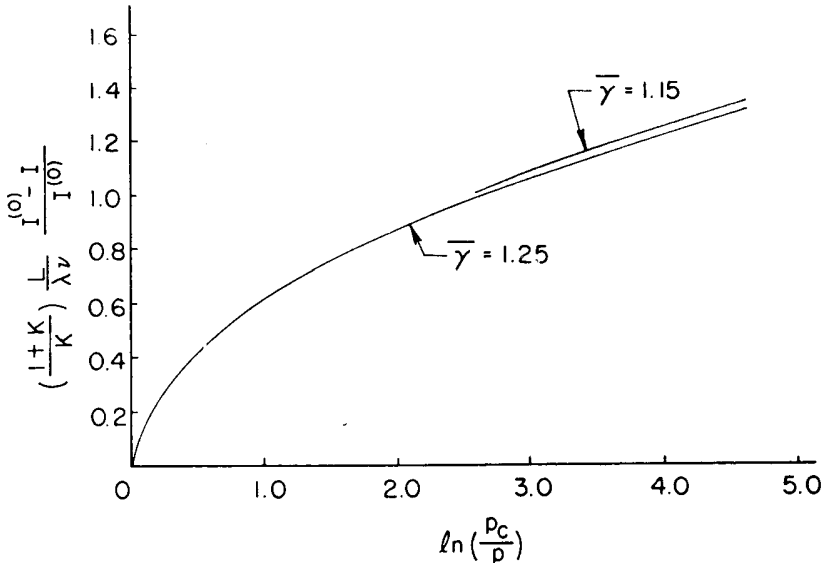

Fig. 7 Fractional loss of specific impulse in nozzle of optimum contour for nozzles of various over-all pressure ratios, $\boldsymbol{p}_{c} / \boldsymbol{p}_{e}(\bar{\gamma}=1.25,1.15 ; \eta=1)$.

areas do. The general elongation of the throat section is shown somewhat more lucidly here than in Fig. 3. The throat occurs somewhat earlier for the optimum contour, but not markedly so. The most pronounced difference is the much more gradual acceleration in the immediate throat area for the nozzle with optimum contour. The contrast between the two is shown more distinctly by comparison of particle slip velocities for the two. Figure 9 shows the ratio of particle slip velocity to chamber sonic velocity in terms of $\ln \left(p_{c} / p\right)$; two items are of particular interest. First, the particle slip velocity in the throat region of the conventional nozzle reaches about three times the value for the nozzle with optimum contour. The peak occurs at the point where the curvature changes, in magnitude and sign, as the flow enters the long exit section. Second, the particle slip at the nozzle exit is much larger for the nozzle with optimum contour than it is for the conventional nozzle. This latter is a direct result of the relatively low accelerations that occur there for conventional nozzles. Moreover, this fact demonstrates the fallacy of judging impulse losses on the basis of particle lag alone. The fact that the particle velocity lag at the nozzle outlet is several times that of a typical conventional nozzle demonstrates that a considerable portion of the impulse loss is associated with dissipation within the nozzle.

The distribution of losses within the two nozzles is most easily shown by calculating the cumulative impulse loss at various stages along the expansion. This can be done by considering Eq. (46) for the impulse loss where the lower limit of the integral is permitted to vary from the chamber pressure toward the prescribed exit pressure. This result then gives the contribution to the impulse loss of all processes taking place up to the pressure corresponding to the lower limit, that is, the cumulative impulse loss to any point of the nozzle. For the optimum nozzle, the similar calculation may be carried out using Eq. (58) in the identical manner.

The results of the cumulative loss calculations are shown in Fig. 10 for both conventional and optimum contours. It is to be noted that the slope of the cumulative loss curve

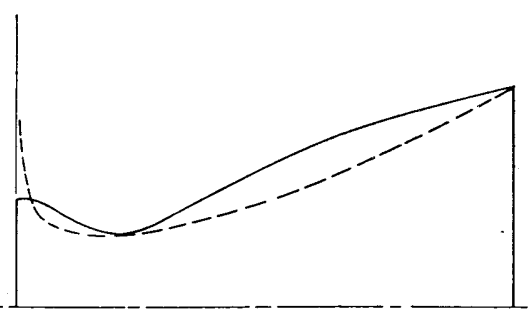

Fig. 8 Comparison of optimum nozzle contour with that of conventional nozzle ${ }^{2}\left(p_{c} / p_{s}=59.6\right)$. 


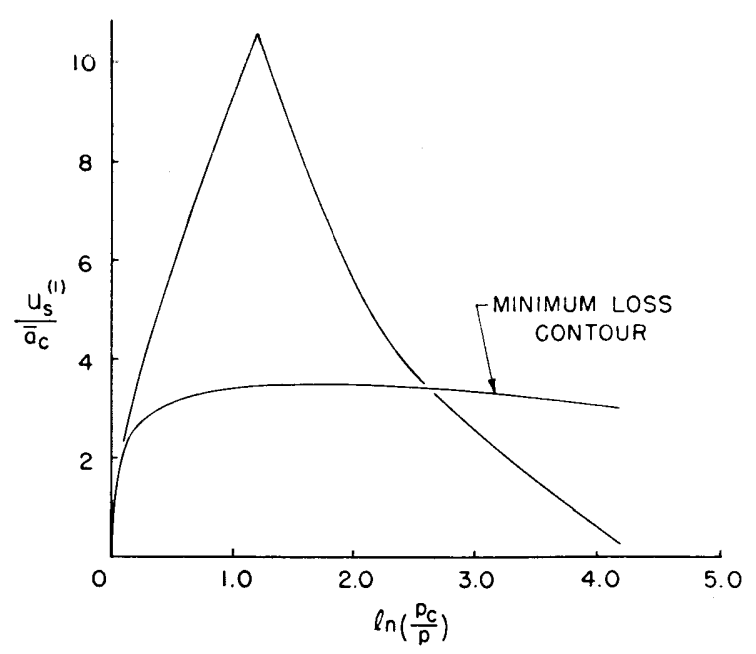

Fig. 9 Comparison of distributions of particle slip velocities for optimum contour and conventional nozzle. 2

bears a close relation to values of a particle slip velocity shown in Fig. 9, confirming the conjecture that dissipation associated with particle slip is a principal contributing factor. The principal increment in loss, which causes the conventional nozzle to result in a lower final impulse than that given by the optimum contour, occurs in the throat region of the conventional nozzle where the particle slip velocities are very high. Similarly, it may be noted that, toward the end of the conventional nozzle, impulse losses are being accumulated very slowly, reflecting the low acceleration rate and particle slip velocities at that area of the conventional nozzle.

In terms of over-all performance, the particle losses in the conventional nozzle appear to be about $40 \%$ larger than those in the optimum nozzle. In a way, this difference may seem relatively small in view of the rather different acceleration profiles of the two. It appears, however, that the losses induced by the particle lag are not an exceedingly sensitive function of the nozzle shape, at least within contour families that would be considered reasonable for the gas alone. As a consequence, it appears that only a minor fraction, e.g., about one-quarter, of the particle-lag loss in a typical conventional nozzle can be regained by use of a nozzle with optimum contour. One may conclude, therefore, that, for practical nozzle shapes, rather small gains in impulse may be expected from more suitable contouring of the nozzle. In terms of current practice, this gain would appear to be a fraction of a percent of the motor impulse.

On the other hand, since the differences between the losses in conventional nozzles and those with optimum contour are not too great, it follows that a reasonable first estimate of the particle loss in a conventional rocket nozzle may be found from the loss in the optimum contour nozzle of the same pressure ratio. This information is contained in the curves of Fig. 7, where, to calculate the fractional impulse loss, it is necessary to know the expansion pressure ratio, the nozzle length, and the mass fraction of solid particles and, finally, to calculate the value of the physical parameter $\lambda_{v}$. It should be mentioned here also that, for particles in excess of $1 \mu$ in radius, or for nozzles of relatively small scale, the slip velocities may be such as to invalidate the Stokes drag law that has been used. These corrections for Reynolds numbers much in excess of unity are not difficult to apply in the manner outlined by Rannie. ${ }^{4}$ For the comparisons shown in Figs. 8-10, taking particles of 5- $\mu$ diam and a nozzle of about 20 -in. length, the average Reynolds number correction to the impulse loss amounts to a factor of $\frac{1}{2}$. That is, the impulse loss is actually about half of that calculated using Stokes law. This result occurs because the particle slip velocity is higher for the lower drag associated with the Stokes law, and consequently the dissipation and direct lag loss are somewhat larger for the Stokes law than for the more accurate drag relationship.

Finally, it should be noted that, for very small particles and large nozzle expansion ratios, the particles enter a gasdynamic slip flow toward the nozzle exit with an attending reduction of drag. Thus, the actual particle speed toward the nozzle exit will fall below that predicted by Stokes law, and the performance will drop slightly. The effect of more accurate particle drag values on the optimum shape will be to make the throat somewhat more abrupt and the exit somewhat more gradual. Although neither of these affects the optimum shape appreciably, it is of interest to note that they both tend toward the conventional nozzle.

\section{Optimum Contour with Fixed Area Ratio}

The analysis can be extended to the case of a nozzle of fixed outlet area rather easily. If there were no particle slip, the pressure $p_{e}{ }^{(0)}$ at the outlet of the nozzle would satisfy the equation

$$
\frac{\rho_{c} \bar{a}_{c} A_{e}}{\dot{m}}=\left(\frac{p_{c}}{p_{c}{ }^{(0)}}\right)^{1 / \bar{\gamma}}\left\{\frac{2}{\bar{\gamma}-1}\left[1-\left(\frac{p_{e}{ }^{(0)}}{p_{c}}\right)^{(\bar{\gamma}-1) / \check{\gamma}}\right]\right\}^{-1 / 2}
$$

Since the outlet area is fixed, particle slip and the attending nonequilibrium effects cause the pressure at the outlet to be modified by an amount proportional to $\bar{\lambda}_{v} / L$. The nozzle outlet pressure takes the form

$$
\frac{p_{c}}{p_{c}}=\frac{p_{e}{ }^{(0)}}{p_{c}}+\frac{\bar{\lambda}_{v}}{L} \frac{p_{c}^{(1)}}{p_{c}}+\ldots
$$

When particle slip occurs, the nozzle outlet area parameter $\left(\rho_{c} \bar{a}_{c} A_{e}\right) / \dot{m}$ may be written to the first order in $\bar{\lambda}_{v} / L$ using Eqs. $(40-42)$, where each of the functions is evaluated at $p_{c} / p_{c}$. But $p_{c} / p_{c}$ is expressed by Eq. (60), so that, retaining only the zeroth-and first-order parts,

$$
\begin{aligned}
& \frac{\rho \bar{a}_{c} A_{e}}{\dot{m}}=\left(\frac{p_{c}}{p_{e}{ }^{(0)}}\right)^{1 / \bar{\gamma}}\left\{\frac{2}{\bar{\gamma}-1}\left[1-\left(\frac{p_{e}{ }^{(0)}}{p_{c}}\right)^{(\bar{\gamma}-1) / \bar{\gamma}}\right]\right\}^{-1 / 2} \times \\
&\left\{1+\frac{\bar{\lambda}_{\gamma}}{L}\left[-\frac{1}{\bar{\gamma}}\left(\frac{p_{c}}{p_{e}(0)}\right)\left(\frac{\bar{\gamma}+1}{2}-\frac{\bar{\gamma}-1}{2}[1-\right.\right.\right. \\
&\left.\left.\left(\frac{p_{e}{ }^{(0)}}{p_{c}}\right)^{(\bar{\gamma}-1) / \bar{\gamma}}\right]^{-1}\right)\left(\frac{p_{e}^{(1)}}{p_{c}}\right)+\frac{\varkappa}{1+\varkappa}([1+ \\
&\left.\left.\left.\left.\frac{1}{(\bar{\gamma}-1) M_{e}{ }^{(0) 2}}\right] G\left(\frac{p_{e}^{(0)}}{p_{c}}\right)-\frac{1}{(\bar{\gamma}-1) M_{e}{ }^{(0) 2}} F\left(\frac{p_{e}^{(0)}}{p_{c}}\right)\right)\right]\right\}
\end{aligned}
$$

The condition that the outlet area parameter be unchanged by the flow perturbation is simply that the nozzle outlet pressure be modified by an amount $\left(\bar{\lambda}_{v} / L\right)\left(p_{c}^{(1)} / p_{c}\right)$ such that

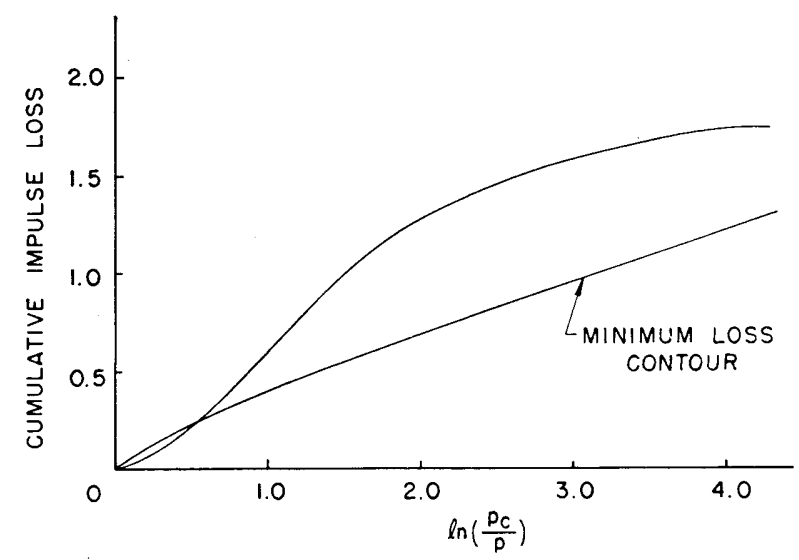

Fig. 10 Comparison of cumulative impulse loss along nozzle of optimum contour with that along conventional nozzle. 
the coefficient of $\bar{\lambda}_{v} / L$ in Eq. (61) vanishes. Explicitly, this gives the outlet pressure perturbation $p_{e}^{(1)} / p_{c}$ as

$$
\frac{p_{e}{ }^{(1)}}{p_{c}}=\frac{\varkappa}{1+\mathcal{x}}\left(\frac{\bar{\gamma} p_{e}{ }^{(0)}}{p_{c}}\right) \frac{\left[1+1 /(\bar{\gamma}-1) M_{e}{ }^{(0) 2}\right] G\left(p_{e}{ }^{(0)} / p_{c}\right)-\left[1 /(\bar{\gamma}-1) M_{e}{ }^{(0) 2}\right] F\left(p_{e}{ }^{(0)} / p_{c}\right)}{(\bar{\gamma}+1) / 2-[(\bar{\gamma}-1) / 2]\left[1-\left(p_{e}{ }^{(0)} / p_{c}\right)^{(\bar{\gamma}-1) / \bar{\gamma}]-1}\right.}
$$

which may then be computed when the functions $F$ and $G$ are known.

Now the thrust of the motor may also be expanded in a perturbation series

$$
\begin{gathered}
\frac{\mathscr{F}^{(0)}+\left(\bar{\lambda}_{v} / L\right) \mathscr{F}^{(1)}}{(1+\varkappa) \dot{m}}=u^{(0)}\left(\frac{p_{e}{ }^{(0)}}{p_{c}}\right)+\frac{d u^{(0)}}{d\left(p / p_{c}\right)} \mid p_{e}{ }^{(0)} \frac{\bar{\lambda}_{v}}{L} \frac{p_{e}{ }^{(1)}}{p_{c}}+ \\
\frac{\lambda_{v}}{L}\left[\frac{1}{1+\varkappa} u^{(1)}\left(\frac{p_{e}^{(0)}}{p_{c}}\right)+\frac{\varkappa}{1+\varkappa} u_{p}^{(1)}\left(\frac{p_{e}{ }^{(0)}}{p_{c}}\right)\right]+ \\
\frac{\bar{\lambda}_{v}}{L} \frac{A_{e} p_{c}}{(1+\varkappa) \dot{m}} \frac{p_{e}^{(1)}}{p_{c}}
\end{gathered}
$$

The straightforward calculation of the derivative shows that

$$
\left.\frac{d u^{(0)}}{d\left(p / p_{c}\right)}\right|_{p_{e}{ }^{(0)}}=-\frac{A_{e} p_{c}}{(1+x) \dot{m}}
$$

and that the remaining perturbation terms may be written as

$$
\begin{aligned}
& -\frac{\bar{\lambda}_{v}}{L}\left(\frac{F^{(1)}}{(1+\varkappa) \dot{m} u^{(0)}}\right) \equiv \frac{I^{(0)}-I}{I^{(0)}}= \\
& \quad-\frac{\bar{\lambda}_{v}}{L}\left[\frac{u^{(1)}}{u^{(0)}}\left(\frac{p_{e}^{(0)}}{p_{c}}\right)-\frac{\varkappa}{1+\varkappa} \frac{u_{s}^{(1)}}{u^{(0)}}\left(\frac{p_{e}^{(0)}}{p_{c}}\right)\right]
\end{aligned}
$$

Comparison with Eq. (44) shows this to be identical with the result obtained for a fixed outlet pressure up to the first-order terms in $\bar{\lambda}_{v} / L$. The variational problem proceeds in exactly the same way as for fixed outlet pressure, and the function $\xi\left(p / p_{c}\right)$ satisfies the same differential equation. The solution is that given in Eqs. (51) and (52), except that the expansion is now carried to the new outlet pressure:

$$
p_{e}=p_{e}{ }^{(0)}+\left(\bar{\lambda}_{v} / L\right) p_{e}{ }^{(1)}
$$

The results of the previous calculations can thus be interpreted to apply also to the optimum nozzle of fixed outlet area. The contour and other properties are identical.

\section{Approximation to the Optimum Solution}

The rather remarkable linearity of $H$ considered as a function of $\ln \left(p_{c} / p\right)$, as shown in Fig. 1, arises from the fact that the factor

$$
\frac{1+\eta(\bar{\gamma}-1) M^{(0) 2}}{M^{(\mathrm{c})}}
$$

in the integral defined by Eq. (52) is nearly constant over the usual range of integration. This factor has a minimum value at the Mach number

$$
M^{(0) *}=[\eta(\bar{\gamma}-1)]^{-1 / 2}
$$

which lies within the range of integration for the usual nozzle Consequently

$$
\frac{1+\eta(\bar{\gamma}-1) M^{(0) 2}}{M^{(0)}} \leqq 2[\eta(\dot{\gamma}-1)]^{1 / 2}
$$

throughout the range of integration and is, in fact, quite close to this value except for pressure ratios close to unity or very far from unity. Therefore, the approximation may be made, from Eq. (52), that

$H\left(\frac{p}{p_{c}}\right) \approx\left[4 \eta(\bar{\gamma}-1)^{1 / 4}\right] \int_{p / p c}^{1} \frac{d \alpha}{\alpha}=$

$$
[4 \eta(\bar{\gamma}-1)]^{1 / 4} \ln \left(\frac{p_{c}}{p}\right)
$$

with the assurance that the exact value of the integral exceeds the approximation. It is clear that this approximate form for the function $H\left(p / p_{c}\right)$ is represented by a straight line through the origin of the logarithmic plot in Fig. 1. Except for rather small pressure ratios $p_{c} / p$, the approximate values of $H\left(p / p_{c}\right)$ fall about $10 \%$ below the exact values and hence constitute a reasonable approximation.

Since the $H\left(p / p_{c}\right)$ function is the only function requiring calculation for the optimum nozzle contour, the foregoing approximation permits all optimum nozzle characteristics to be expressed in very simple forms. Of particular interest here is the pressure distribution

$$
\xi\left(p / p_{c}\right) \approx \ln \left(p_{c} / p\right)
$$

which may be compared with the exact value in Fig. 2, and the fractional specific impulse loss

$$
\frac{I^{(0)}-I}{I^{(0)}} \approx \frac{\lambda}{L}\left(\frac{\varkappa}{1+x}\right) \cdot 2[\eta(\bar{\gamma}-1)]^{1 / 2}\left\{\frac{\ln \left(p_{c} / p_{e}\right)}{\gamma M_{e}(0)}\right\}^{2}
$$

This last expression is to be compared with the exact calculations of Fig. 7; the approximate values are not more than $20 \%$ below the exact ones.

Finally, referring to Eq. (41), it is possible to express the optimum nozzle area as an explicit function of distance measured from the chamber as

$\frac{\rho_{c} \bar{a}_{c} A^{(0)}}{\dot{m}} \approx \frac{1}{2}[\bar{\gamma}-1]^{1 / 2} e^{[(\bar{\gamma}+1) / 2 \bar{\gamma}] \xi}\left[\operatorname{csch}\left(\frac{\bar{\gamma}-1}{2 \bar{\gamma}} \xi\right)\right]^{1 / 2}$

which provides a convenient and reasonably accurate representation of the nozzle shape.

\section{Concluding Remarks}

Probably the most interesting result of the foregoing analysis is that the difference between the particle lag loss in a conventional nozzle and that in the corresponding optimum nozzle is not very large in spite of the fact that rather large velocity and temperature lags may occur near the throat of the conventional nozzle. Consequently, it is usually unwarranted to construct exotic contours since this effort, carried out at the expense of many other features of the nozzle, will not reduce the particle loss by more than one-third. This is not to imply that improvement in existing nozzles cannot be made, but rather that the gains will be small unless the original nozzle was very short, or very small scale, or possessed some unusual entrance condition or throat contour.

As a consequence, it follows that the performance of a conventional nozzle may be approximated by the performance of the optimum nozzle having the same pressure ratio or area ratio. Often this rather modest inaccuracy is more than compensated for by avoiding the numerical calculation required for an arbitrary contour. The error made in this approximation is usually less than that resulting from inadequate knowledge of the particle size, gas properties, etc. In fact, the elementary expressions developed in the latter part of the paper, Eqs. (70-72), will suffice for rough estimates of the usual sort.

The major approximation in the present analysis lies in the use of Stokes law and the corresponding approximation for the heat transfer from particles. For particles of $5-\mu$ diam in a nozzle of 20 -in. length, the present analysis predicts absolute losses that are high by a factor of nearly two. This error diminishes very rapidly with particle size, becom- 
ing negligible for 2- $\mu$-diam particles. Errors of similar magnitude may be incurred due to slip flow.

\section{References}

${ }^{1}$ Gilbert, M., Davis, L., and Altman, D., "Velocity lag of particles in linearly accelerated combustion gases," Jet Propulsion 25, 25-30 (1955).

2 Kliegel, J. R., "One-dimensional flow of a gas-particle system," IAS Paper 60-3 (January 1960).
${ }^{3}$ Hogland, R. F., "Recent advances in gas particle nozzle flows," ARS J. 32, 662-671 (1962).

${ }^{4}$ Rannie, W. D., "A perturbation analysis of one-dimensional heterogeneous flow in rocket nozzles," Progress in Astronautics and Rocketry: Detonation and Two-Phase Flow (Academic Press, New York, 1962); Vol. 6.

${ }^{5}$ Marble, F. E., "Dynamics of a gas containing small solid particles," Proceedings of the Fifth AGARD Combustion and Propulsion Colloquium (Pergamon Press, New York, 1963). 